\title{
A scheme for protecting one-qubit information against erasure error
}

\author{
Chui-Ping Yang ${ }^{1}$, Shih-I Chu ${ }^{1}$, and Siyuan Han ${ }^{2}$ \\ ${ }^{1}$ Department of Chemistry, University of Kansas, and Kansas Center \\ for Advanced Scientific Computing, Lawrence, Kansas 66045, USA \\ ${ }^{2}$ Department of Physics and Astronomy, University of Kansas, Lawrence, \\ Kansas 66045, USA
}

\begin{abstract}
We propose a scheme for protecting one qubit information against one erasure. The scheme operates essentially by distributing quantum information over four qubits. Two versions of the method are considered: one of them is based on two spatially-separated pairs of qubits, and the other is based on one pair of qubits and two spatially-separated qubits.
\end{abstract}

PACS numbers: 03.67.Lx, 03.65.Bz

Typeset using REVTEX 
Quantum computing has become an active aspect of current research fields with the discovery of Shor's algorithm for factorizing a large number [1-2], though the fact that quantum computer might, in principle, allow to solve hard computational problems more efficiently than their classical counterpart is, at present, still a conjecture to a large extent [3]. It is known that the biggest difficulty inhibiting realizations of quantum computers is the fragility of quantum states. Decoherence of qubits caused by the interaction with environment will collapse the state of the quantum computer and thus lead to the loss of information. To solve this problem, many new QECCs have been discovered [4-19]. For a sufficiently weak noise environment, Knill and Laflamme have shown that a five qubit code, the smallest quantum error correction code, can be applied to encode one qubit of quantum information and to correct a single-qubit arbitrary error at an unknown position [6].

In 1997 M. Grassl et al. [20] considered an error model where the position of the erroneous qubits is known. In accordance with classical coding theory, they called this model the quantum erasure channel. Some physical scenarios to determine the position of an error have been given [20]. In their work, they showed that only four-qubit error correction code is required to encode one qubit and correct one erasure ( i.e., a single-qubit arbitrary error for which the position of the "damaged" qubit is known). Clearly, this code is very important for protecting one qubit of quantum information as long as the position of the "bad" qubit is known. In this paper, we propose an alternate scheme and some comparison with Ref. $[20]$ will be given in the following.

We first discuss how to protect one qubit information against one erasure based on two spatially-separated pairs of qubits. Each pair contains two qubits which are set close in a sense that environment cannot tell them apart, i.e., the two qubits in each pair have a good symmetry that they will undergo collective decoherence. We denote the two qubits in one pair by 1 and $1^{\prime}$ while the two qubits in the other pair by 2 and $2^{\prime}$. The qubit 1 is the original information carrier, which may be in a general state

$$
\alpha|0\rangle+\beta|1\rangle .
$$


The basis states for our code are shown as follows

$$
\begin{aligned}
|0\rangle_{L} & =|01\rangle_{11^{\prime}}|01\rangle_{22^{\prime}}, \\
|1\rangle_{L} & =|10\rangle_{11^{\prime}}|10\rangle_{22^{\prime}} .
\end{aligned}
$$

The encoding equations (2) can be implemented by the quantum CNOT (controlledNOT) operations $C_{i j}$, where the first subscript of $C_{i j}$ refers to the control bit and the second to the target. The three ancillary qubits $1^{\prime}, 2$ and $2^{\prime}$ are initially in the state $|100\rangle$. Throughout this paper, every joint operation will follow the sequence from right to left. Let a joint encoding operation $C_{1^{\prime} 2^{\prime}} C_{12} C_{11^{\prime}}$ act on the four qubits, then the state (1) will be encoded into

$$
|\psi\rangle_{L}=\alpha|01\rangle_{11^{\prime}}|01\rangle_{22^{\prime}}+\beta|10\rangle_{11^{\prime}}|10\rangle_{22^{\prime}}
$$

Depending on the application envisioned, the "encoded state" (3) could be used as is (since it has been shown that one can construct logical gates that work on "encoded" qubits of this form [21]), or one could factor out the ancillary qubits, as needed, by applying one more time the same CNOT operation.

Let us first give the interpretations of the encoding (2) in terms of error correction codes. It is easily seen that no matter which pair goes "bad", both of the logical states (2) satisfy the erasure-correcting conditions $[20,22]$

$$
\begin{aligned}
& \left\langle i_{L}\left|A_{a}\right| i_{L}\right\rangle=\left\langle j_{L}\left|A_{a}\right| j_{L}\right\rangle, \\
& \left\langle i_{L}\left|A_{a}\right| j_{L}\right\rangle=0 \quad \text { for }\left\langle i_{L} \mid j_{L}\right\rangle=0 .
\end{aligned}
$$

with $i, j=0,1$, and $A_{a}$ are the error operators for the "bad" pair, which are any linear combinations and products of the algebra basis $\left\{1, \sum_{i=1}^{1^{\prime}} \sigma_{i x}, \sum_{i=1}^{1^{\prime}} \sigma_{i y}, \sum_{i=1}^{1^{\prime}} \sigma_{i z}\right\}$ or $\left\{1, \sum_{i=2}^{2^{\prime}} \sigma_{i x}, \sum_{i=2}^{2^{\prime}} \sigma_{i y}, \sum_{i=2}^{2^{\prime}} \sigma_{i z}\right\}$. Thus, both logical states in (2) can be regarded as an erasurecorrecting code: it can, in principle, encode one qubit and correct one pair erasure. In the following, we will show explicitly how this can be done. 
Suppose that the environment is in a mixed state $\rho_{0}=\sum_{i} p_{i}|\epsilon\rangle_{i}\langle\epsilon|$ (where $\rho_{0}$ is a density operator of the environment and $p_{i}$ is a probability with which the environment is in the pure state $|\epsilon\rangle_{i}$ ), then the state of the whole system composed of the qubit system and the environment is describled by

$$
\rho_{L} \otimes \rho_{0}=\sum_{i} p_{i}|\psi\rangle_{L}|\epsilon\rangle_{i}\left\langle\psi | _ { L } \left\langle\left.\epsilon\right|_{i}\right.\right.
$$

where $\rho_{L}=|\psi\rangle_{L}\left\langle\left.\psi\right|_{L}\right.$ which is a density operator of the encoded qubit system. It follows from equation (3) that

$$
\begin{array}{r}
\rho_{L} \otimes \rho_{0}=\sum_{i} p_{i}\left(\alpha|01\rangle_{11^{\prime}}|01\rangle_{22^{\prime}}+\beta|10\rangle_{11^{\prime}}|10\rangle_{22^{\prime}}\right)|\epsilon\rangle_{i} \\
\otimes\left(\alpha ^ { * } \left\langle0 1 | _ { 1 1 ^ { \prime } } \left\langle\left.01\right|_{22^{\prime}}+\beta^{*}\left\langle1 0 | _ { 1 1 ^ { \prime } } \langle 1 0 | _ { 2 2 ^ { \prime } } ) \left\langle\left.\epsilon\right|_{i} .\right.\right.\right.\right.\right.
\end{array}
$$

One can certainly envision situations where one might, in fact, know which qubit pair goes bad while the other pair is intact (by using the methods for determining the position of an error [20]). Also, we assume that collective decoherence holds exactly for each separate pair. In this case, the most general kind of error that could affect a qubit pair can be expressed in the form

$$
\begin{aligned}
|01\rangle|\epsilon\rangle_{i} & \rightarrow|00\rangle\left|\epsilon_{0}\right\rangle_{i}+|01\rangle\left|\epsilon_{1}\right\rangle_{i}+|10\rangle\left|\epsilon_{2}\right\rangle_{i}+|11\rangle\left|\epsilon_{3}\right\rangle_{i} \\
|10\rangle|\epsilon\rangle_{i} & \rightarrow|00\rangle\left|\epsilon_{0}\right\rangle_{i}+|10\rangle\left|\epsilon_{1}\right\rangle_{i}+|01\rangle\left|\epsilon_{2}\right\rangle_{i}+|11\rangle\left|\epsilon_{3}\right\rangle_{i},
\end{aligned}
$$

(assuming that the system does not actually leave the computational space), where $\left|\epsilon_{j}\right\rangle_{i}$ are appropriate environment states, not necessarily orthogonal or normalized. The collective decoherence condition is expressed by the equality of the environment states appearing in (7a) and (7b): the fact that the environment "cannot tell the qubits apart" means that the evolution of the state $\left|i i^{\prime}\right\rangle$ is obtained from that of the state $\left|i^{\prime} i\right\rangle$ by merely exchanging the two qubit values everywhere. Equivalently, the conditions for collective noise behavior of (7a)-(7b) can be obtained by evolving the logical states under the interaction algebra formed by totally symmetric operators on the relevant pairs (in the language of Ref. [23]). 
Consider first the case in which the first pair $\left(1,1^{\prime}\right)$ in $(3)$ undergoes decoherence of the form (7). The resulting state is

$$
\rho=\sum_{i} p_{i}|\psi\rangle_{i}\langle\psi|
$$

where

$$
\begin{aligned}
|\psi\rangle_{i}=\alpha & \left(|00\rangle_{11^{\prime}}\left|\epsilon_{0}\right\rangle_{i}+|01\rangle_{11^{\prime}}\left|\epsilon_{1}\right\rangle_{i}+|10\rangle_{11^{\prime}}\left|\epsilon_{2}\right\rangle_{i}+|11\rangle_{11^{\prime}}\left|\epsilon_{3}\right\rangle_{i}\right)|01\rangle_{22^{\prime}} \\
& +\beta\left(|00\rangle_{11^{\prime}}\left|\epsilon_{0}\right\rangle_{i}+|10\rangle_{11^{\prime}}\left|\epsilon_{1}\right\rangle_{i}+|01\rangle_{11^{\prime}}\left|\epsilon_{2}\right\rangle_{i}+|11\rangle_{11^{\prime}}\left|\epsilon_{3}\right\rangle_{i}\right)|10\rangle_{22^{\prime}}
\end{aligned}
$$

To disentangle the first pair from the second pair in (9), do first a CNOT operation $C_{1^{\prime} 1}$ on it, to get

$$
\rho=\sum_{i} p_{i}\left|\psi^{\prime}\right\rangle_{i}\left\langle\psi^{\prime}\right|
$$

where

$$
\begin{aligned}
\left|\psi^{\prime}\right\rangle_{i}=\alpha & {\left[|0\rangle_{1}\left(|0\rangle_{1^{\prime}}\left|\epsilon_{0}\right\rangle_{i}+|1\rangle_{1^{\prime}}\left|\epsilon_{3}\right\rangle_{i}\right)+|1\rangle_{1}\left(|1\rangle_{1^{\prime}}\left|\epsilon_{1}\right\rangle_{i}+|0\rangle_{1^{\prime}}\left|\epsilon_{2}\right\rangle_{i}\right)\right]|01\rangle_{22^{\prime}} } \\
& +\beta\left[|0\rangle_{1}\left(|0\rangle_{1^{\prime}}\left|\epsilon_{0}\right\rangle_{i}+|1\rangle_{1^{\prime}}\left|\epsilon_{3}\right\rangle_{i}\right)+|1\rangle_{1}\left(|0\rangle_{1^{\prime}}\left|\epsilon_{1}\right\rangle_{i}+|1\rangle_{1^{\prime}}\left|\epsilon_{2}\right\rangle_{i}\right)\right]|10\rangle_{22^{\prime}}
\end{aligned}
$$

and then measure the state of qubit 1 . If the result is zero, the qubit $1^{\prime}$ factors out, and thus, the second pair with the environment and the qubit $1^{\prime}$ will be in the state

$$
\begin{aligned}
\rho= & {\left[\sum _ { i } p _ { i } ( | 0 \rangle _ { 1 ^ { \prime } } | \epsilon _ { 0 } \rangle _ { i } + | 1 \rangle _ { 1 ^ { \prime } } | \epsilon _ { 3 } \rangle _ { i } ) \left(\left\langle0 | _ { 1 ^ { \prime } } \left\langle\left.\epsilon_{0}\right|_{i}+\left\langle\left. 1\right|_{1^{\prime}}\left\langle\left.\epsilon_{3}\right|_{i}\right)\right] \otimes\right.\right.\right.\right.} \\
& \left(\alpha|01\rangle_{22^{\prime}}+\beta|10\rangle_{22^{\prime}}\right)\left(\alpha ^ { * } \left\langle\left.01\right|_{22^{\prime}}+\beta^{*}\left\langle\left. 10\right|_{22^{\prime}}\right) .\right.\right.
\end{aligned}
$$

Equation (12) implies that the pair $\left(2,2^{\prime}\right)$ is disentangled from the environment and the qubit $1^{\prime}$, and it is automatically in the coherent superposition

$$
\alpha|01\rangle_{22^{\prime}}+\beta|10\rangle_{22^{\prime}}
$$

On the other hand, if the result is one, all one has to do is flip qubit $1^{\prime}$ conditioned on the state of the remaining pair; this can be accomplished, for instance, using a gate $C_{21^{\prime}}$ (after the operation $C_{21^{\prime}}$, qubit $1^{\prime}$ will factor out and the second pair $\left(2,2^{\prime}\right)$ will be in the state 
(13)). Finally, we perform an operation $C_{22^{\prime}}$, then, it follows from (13) that the original state (1) will be recovered through the qubit 2.

Note that for each logical state in the encoding (2), the qubits $(1,2)$ and the qubits $\left(1^{\prime}, 2^{\prime}\right)$ are in the same states. Thus, if the second pair goes bad, the original state can be restored through the qubit 1 by following above procedures.

In what follows, we will discuss how to protect one qubit information against one erasure based on a qubit pair and two spatially-separated qubits. As described above, the two qubits in the pair are set close so that they have a good symmetry, and therefore, they will undergo collective decoherence. We denote the two qubits in the pair by 1 and $1^{\prime}$ while the other two separate qubits by 2 and $2^{\prime}$. We still assume that qubit 1 is the original information carrier. Our encoding is shown as follows

$$
\begin{aligned}
|0\rangle_{L} & =\frac{1}{\sqrt{2}}|01\rangle_{11^{\prime}}(|00\rangle+|11\rangle)_{22^{\prime}}, \\
|1\rangle_{L} & =\frac{1}{\sqrt{2}}|10\rangle_{11^{\prime}}(|00\rangle-|11\rangle)_{22^{\prime}},
\end{aligned}
$$

which can be done: the ancillary three qubits are first prepared in the state $|100\rangle_{1^{\prime} 22^{\prime}}$, then we perform a joint operation $C_{11^{\prime}} C_{22^{\prime}} H_{2} C_{12}$ on the qubit system where $H_{2}$ is a Hadamard transformation on qubit 2 , sending $|0\rangle \rightarrow(|0\rangle+|1\rangle) / \sqrt{2}$ and $|1\rangle \rightarrow(|0\rangle-|1\rangle) / \sqrt{2}$. The encoded state will be

$$
|\psi\rangle_{L}=\alpha \frac{1}{\sqrt{2}}|01\rangle_{11^{\prime}}(|00\rangle+|11\rangle)_{22^{\prime}}+\beta \frac{1}{\sqrt{2}}|10\rangle_{11^{\prime}}(|00\rangle-|11\rangle)_{22^{\prime}}
$$

Thus, from (5) and (15) the state of the qubit system and the environment is given by

$$
\begin{aligned}
\rho_{L} \otimes \rho_{0}= & \sum_{i} p_{i}\left(\alpha \frac{1}{\sqrt{2}}|01\rangle_{11^{\prime}}(|00\rangle+|11\rangle)_{22^{\prime}}+\beta \frac{1}{\sqrt{2}}|10\rangle_{11^{\prime}}(|00\rangle-|11\rangle)_{22^{\prime}}\right)|\epsilon\rangle_{i} \\
& \otimes\left(\alpha ^ { * } \frac { 1 } { \sqrt { 2 } } \left\langle\left.01\right|_{11^{\prime}}(\langle 00|+\langle 11|)_{22^{\prime}}+\beta^{*} \frac{1}{\sqrt{2}}\left\langle\left. 10\right|_{11^{\prime}}(\langle 00|-\langle 11|)_{22^{\prime}}\right)\left\langle\left.\epsilon\right|_{i} .\right.\right.\right.
\end{aligned}
$$

Consider first the case in which the pair $\left(1,1^{\prime}\right)$ undergoes collective decoherence of the form (7). The resulting state is

$$
\rho=\sum_{i} p_{i}|\psi\rangle_{i}\langle\psi|
$$


where

$$
\begin{aligned}
|\psi\rangle_{i}=\frac{1}{\sqrt{2}} \alpha\left(|00\rangle_{11^{\prime}}\left|\epsilon_{0}\right\rangle_{i}+|01\rangle_{11^{\prime}}\left|\epsilon_{1}\right\rangle_{i}+|10\rangle_{11^{\prime}}\left|\epsilon_{2}\right\rangle_{i}+|11\rangle_{11^{\prime}}\left|\epsilon_{3}\right\rangle_{i}\right)(|00\rangle+|11\rangle)_{22^{\prime}} \\
+\frac{1}{\sqrt{2}} \beta\left(|00\rangle_{11^{\prime}}\left|\epsilon_{0}\right\rangle_{i}+|10\rangle_{11^{\prime}}\left|\epsilon_{1}\right\rangle_{i}+|01\rangle_{11^{\prime}}\left|\epsilon_{2}\right\rangle_{i}+|11\rangle_{11^{\prime}}\left|\epsilon_{3}\right\rangle_{i}\right)(|00\rangle-|11\rangle)_{22^{\prime}} .
\end{aligned}
$$

What we need to do now is to disentangle the pair from the other two qubits $\left(2,2^{\prime}\right)$ in $(18)$. We first perform a joint operation $H_{2} C_{22^{\prime}} C_{1^{\prime} 1}$ which leads to

$$
\rho=\sum_{i} p_{i}\left|\psi^{\prime}\right\rangle_{i}\left\langle\psi^{\prime}\right|
$$

where

$$
\begin{aligned}
\left|\psi^{\prime}\right\rangle_{i}=\alpha & {\left[|0\rangle_{1}\left(|0\rangle_{1^{\prime}}\left|\epsilon_{0}\right\rangle_{i}+|1\rangle_{1^{\prime}}\left|\epsilon_{3}\right\rangle_{i}\right)+|1\rangle_{1}\left(|1\rangle_{1^{\prime}}\left|\epsilon_{1}\right\rangle_{i}+|0\rangle_{1^{\prime}}\left|\epsilon_{2}\right\rangle_{i}\right)\right]|00\rangle_{22^{\prime}} } \\
& +\beta\left[|0\rangle_{1}\left(|0\rangle_{1^{\prime}}\left|\epsilon_{0}\right\rangle_{i}+|1\rangle_{1^{\prime}}\left|\epsilon_{3}\right\rangle_{i}\right)+|1\rangle_{1}\left(|0\rangle_{1^{\prime}}\left|\epsilon_{1}\right\rangle_{i}+|1\rangle_{1^{\prime}}\left|\epsilon_{2}\right\rangle_{i}\right)\right]|11\rangle_{22^{\prime}}
\end{aligned}
$$

and then we measure the state of qubit 1 . If the result is zero, the qubit $1^{\prime}$ factors out, and thus, the two qubits $\left(2,2^{\prime}\right)$ with the environment and the qubit $1^{\prime}$ will be in the state

$$
\begin{aligned}
\rho= & {\left[\sum _ { i } p _ { i } ( | 0 \rangle _ { 1 ^ { \prime } } | \epsilon _ { 0 } \rangle _ { i } + | 1 \rangle _ { 1 ^ { \prime } } | \epsilon _ { 3 } \rangle _ { i } ) \left(\left\langle0 | _ { 1 ^ { \prime } } \left\langle\left.\epsilon_{0}\right|_{i}+\left\langle\left. 1\right|_{1^{\prime}}\left\langle\left.\epsilon_{3}\right|_{i}\right)\right]\right.\right.\right.\right.} \\
& \otimes\left(\alpha|00\rangle_{22^{\prime}}+\beta|11\rangle_{22^{\prime}}\right)\left(\alpha ^ { * } \left\langle\left.00\right|_{22^{\prime}}+\beta^{*}\left\langle\left. 11\right|_{22^{\prime}}\right) .\right.\right.
\end{aligned}
$$

Equation (21) shows that the pair $\left(2,2^{\prime}\right)$ is disentangled from the environment and the qubit $1^{\prime}$, and it is automatically in the coherent superposition

$$
\alpha|00\rangle_{22^{\prime}}+\beta|11\rangle_{22^{\prime}}
$$

On the other hand, if the result is one, one has to flip qubit $1^{\prime}$ by performing a gate operation $C_{21^{\prime}}$. From Equation (22), an operation $C_{22^{\prime}}$ will lead to recovery of the original state (1) through qubit 2.

The encoding (14) is invariant under the exchange of the bit values for the qubits 2 and $2^{\prime}$. Thus, if the original state can be recovered after the qubit 2 goes bad, it can also be recovered in a similar way for the case of the qubit $2^{\prime}$ going bad. To simplify our presentation, however, we will only discuss how to restore the original state when the qubit 2 goes bad. 
Since $|0\rangle$ and $|1\rangle$ form a basis for a qubit, we need only know what happens to these two states. In general, the single-qubit decoherence process must be

$$
\begin{aligned}
|0\rangle|\epsilon\rangle_{i} & \rightarrow|0\rangle\left|\epsilon_{0}\right\rangle_{i}+|1\rangle\left|\epsilon_{1}\right\rangle_{i}, \\
|1\rangle|\epsilon\rangle_{i} & \rightarrow|0\rangle\left|\epsilon_{0}^{\prime}\right\rangle_{i}+|1\rangle\left|\epsilon_{1}^{\prime}\right\rangle_{i} .
\end{aligned}
$$

As will be shown below, during the restoration operation there is no need of performing any operations on the "damaged" qubit. For the simplicity, we can rewrite Eq. (23) as

$$
\begin{aligned}
|0\rangle|\epsilon\rangle_{i} & \rightarrow\left|\widetilde{0}_{i}\right\rangle, \\
|1\rangle|\epsilon\rangle_{i} & \rightarrow\left|\widetilde{1}_{i}\right\rangle,
\end{aligned}
$$

where the above environment states $\left|\epsilon_{0}\right\rangle_{i},\left|\epsilon_{1}\right\rangle_{i},\left|\epsilon_{0}^{\prime}\right\rangle_{i}$ and $\left|\epsilon_{1}^{\prime}\right\rangle_{i}$ have been included in $|\widetilde{0}\rangle_{i}$ and $|\widetilde{1}\rangle_{i}$. Let us now see what will happen to the state (16). After the qubit 2 goes bad, it goes to

$$
\rho=\sum_{i} p_{i}|\phi\rangle_{i}\langle\phi|
$$

where

$$
|\phi\rangle_{i}=\frac{1}{\sqrt{2}}\left[\left(\alpha|01\rangle_{11^{\prime}}+\beta|10\rangle_{11^{\prime}}\right)\left|\widetilde{0}_{i} 0\right\rangle_{22^{\prime}}+\left(\alpha|01\rangle_{11^{\prime}}-\beta|10\rangle_{11^{\prime}}\right)\left|\widetilde{1}_{i} 1\right\rangle_{22^{\prime}}\right]
$$

A phase-shift gate operation $P_{2^{\prime} 1^{\prime}}$ on the qubits $2^{\prime}$ and $1^{\prime}$, which sends $|i j\rangle \rightarrow(-1)^{j \times j}|i j\rangle($ here, $i, j \in\{0,1\})$, leads Equation (26) to

$$
\frac{1}{\sqrt{2}}\left(\alpha|01\rangle_{11^{\prime}}+\beta|10\rangle_{11^{\prime}}\right)\left(\left|\widetilde{0}_{i} 0\right\rangle_{22^{\prime}}-\left|\widetilde{1}_{i} 1\right\rangle_{22^{\prime}}\right) \text {. }
$$

It follows from Equations (25) and (27) that the state of the qubit system and the environment is now given by

$$
\begin{aligned}
\rho= & {\left[\frac { 1 } { 2 } \sum _ { i } p _ { i } ( | \widetilde { 0 } _ { i } 0 \rangle _ { 2 2 ^ { \prime } } - | \widetilde { 1 } _ { i } 1 \rangle _ { 2 2 ^ { \prime } } ) \left(\left\langle\left.\widetilde{0}_{i} 0\right|_{22^{\prime}}-\left\langle\left.\widetilde{1}_{i} 1\right|_{22^{\prime}}\right)\right]\right.\right.} \\
& \otimes\left(\alpha|01\rangle_{11^{\prime}}+\beta|10\rangle_{11^{\prime}}\right)\left(\alpha ^ { * } \left\langle\left.01\right|_{11^{\prime}}+\beta^{*}\left\langle\left. 10\right|_{11^{\prime}}\right) .\right.\right.
\end{aligned}
$$

Equation (28) implies that after a simple phase-shift operation, the pair (1, 1') has been disentangled from the other two qubits and the environment; and it is automatically in the 
coherent superposition $\alpha|01\rangle_{11^{\prime}}+\beta|10\rangle_{11^{\prime}}$, from which the original state (1) can be recovered through the qubit 1 after performing a CNOT operation $C_{11^{\prime}}$.

The above procedures show that during the restoration operation, the "damaged" qubit 2 is not involved at all and there is no need of performing a measurement on any qubit (the same situation also holds for the case of the qubit $2^{\prime}$ going bad).

As shown above, some of the qubits have to be set close so that they undergo collective decoherence; while the scheme of Grassl et al [20], each qubit undergoes independent decoherence, i.e., any two of the four qubits are spatially separated. In this sense, their scheme has a loosen condition and thus it is better than ours. However, like our scheme, their scheme works in the case when the error position is known, i.e, one has to detect over each qubit to determine which qubit goes bad (for example, if errors are accompanied by the emission of quanta, they can in principle be detected). Thus, in their scheme, one has to detect over four spatially-separated qubits at the same time; while in our scheme the detection is simpler, for instance, we need only two detectors to detect over two separate pairs for the first version of the method.

Finally, it should be made more clearer that, in general, ensuring the conditions for collective error behavior requires more than setting qubits sufficiently close. For instance, the no-qubits-interaction condition should be satisfied in the collective decoherence models [24]. Another related point is that experimental realizations of collective noise in qubit systems have been obtained to date by using trapped ions [25] and liquid-state NMR [26,27].

In summary, we have presented an alternate scheme to protect one qubit information against one erasure error, by using imperfect symmetry. The encoding and error recovery operations, as shown here, are relatively straightforward. A special feature of the present scheme is that it requires less detection.

\section{ACKNOWLEDGMENTS}

This work was partially supported by National Science Foundation (EIA-0082499). 


\section{REFERENCES}

[1] Shor P W in Proc. 35th Annual Symp. on Foundations of Computer Science ( IEEE Computer Society Press, New York 1994), pp. 124-134

[2] Chuang I L, Laflamme R, Shor P W and Zurek W H 1995 Science 2701633

[3] Laflamme R et al. 2002 Quantum Inf. \& Comp. 2166

[4] Shor P W 1995 Phys. Rev. A 52 R2493

[5] Steane A M 1996 Phys. Rev. Lett. 77793

[6] Laflamme R, Miquel C, Paz J P and Zurek W H 1996 Phys. Rev. Lett. 77198

[7] Ekert A and Macchiavello C 1996 Phys. Rev. Lett. 772585

[8] Gottesman D 1996 Phys. Rev. A 541862

[9] Bennett C H, DiVincenzo D P, Smolin J A and Wootters W K 1996 Phys. Rev. A 54 3824

[10] Steane A M 1996 Phys. Rev. A 54 4741; Steane A M 1996 Proc. R. Soc. London A 452 2551

[11] Shor P W 1996 LANL preprint quant-ph/9605011; Shor P and Laflamme R 1997 Phys. Rev. Lett. 781600

[12] DiVincenzo D P and Shor P W 1996 Phys. Rev. Lett. 773260

[13] Zurek W H and Laflamme R 1996 Phys. Rev. Lett. 774683

[14] Plenio M B, Vedral V and Knight P L 1997 Phys. Rev. A 5567

[15] Calderbank A R, Rains E M, Shor P W and Sloane N J A 1997 Phys. Rev. Lett. 78 405

[16] Knill E and Laflamme R 1997 Phys. Rev. A 55900 
[17] Leung D W, Nielsen M A, Chuang I L and Yamamoto Y 1997 Phys. Rev. A 562567

[18] Preskill J 1997 LANL preprint quant-ph/9705031

[19] Bennett C H and Shor P W 1998 IEEE Trans. Inf. Theory 442724

[20] Grassl M, Beth Th and Pellizzari T 1997 Phys. Rev. A 5633

[21] Bacon D, Kempe J, Lidar D A and Whaley K B 2000 Phys. Rev. Lett. 851758

[22] Cerf N J and Cleve R 1997 Phys. Rev. A 561721

[23] Knill E, Laflamme R and Viola L 2000 Phys. Rev. Lett. 842525

[24] Hwang W Y, Ahn D D, and Hwang S W 2001 Phys. Rev. A 63022303

[25] Kielpinski D, Meyer V, Rowe M A, Sackett C A, Itano W M, Monroe C, and Wineland

D J 2001 Science 2911013

[26] Viola L, Fortunato E M, Pravia M A, Knill E, Laflamme R, and Coryet D G 2001 Science 2932059

[27] Fortunato E M, Viola L, Hodges J, Teklemariam G and Cory D G 2002 New J. Phys. 4 5.1-5.20

[28] Knill E, Laflamme R, Martinez R and Negrevergne C 2001 Phys. Rev. Lett. 865811 